\title{
A Modular Multilevel Converter with Isolated Energy-Balancing Modules for MV Drives Incorporating Symmetrical Six-Phase Machines
}

\author{
Mohamed S. Diab, B. W. Williams, Derrick Holliday \\ Electronic and Electrical Engineering Dept. \\ University of Strathclyde \\ Glasgow, UK
}

\author{
Ahmed M. Massoud \\ Electrical Engineering Dept. \\ Qatar University \\ Doha, Qatar
}

\author{
Shehab Ahmed \\ ECEN Dept. \\ Texas A\&M University at Qatar \\ Doha, Qatar
}

\begin{abstract}
A modular multilevel converter (MMC) configuration is presented as a medium-voltage high-power drive for variablespeed applications incorporating symmetrical six-phase machines. The topology employs six standard MMC phase-legs feeding two isolated groups of three-phase machine windings, each with $60^{\circ}$ spatial phase-displacement. A novel concept of ripple-power decoupling between adjacent sub-modules (SMs) of MMC phaselegs, while feeding machine windings in a spatial phase-opposition fashion, is realized through isolated dc-dc converter modules. This concept offers a significant reduction in SM capacitance, while eliminating the problem of SM capacitor wide voltage fluctuations, especially at low operating frequencies. Furthermore, the proposed configuration can efficiently operate at near zero frequency, therefore a machine speed-range from zero speed up to the rated speed is possible under rated torque operating conditions. The proposed MMC configuration is analyzed in detail, and its performance is validated using both simulation and experimentation.
\end{abstract}

Index Terms-Dual half-bridge (DHB), medium-voltage variable-speed drives, modular multilevel converter (MMC), power decoupling, sub-module (SM) capacitor voltage-ripple, symmetrical six-phase machines.

\section{INTRODUCTION}

Interest in medium-voltage (MV) variable-speed drives has grown such that they are now deployed in high-power applications in various industrial sectors, triggering the development of MV converter topologies able to empower industrial drives at the megawatt range [1]. Among variablespeed drives, applications incorporating three-phase machines have been the focus of attention. Nonetheless, higher phasenumber machines have been rejuvenated due to the flexibility of realizing any number of phase voltages using dc-ac converters. Multiphase machines have unique advantages over their three-phase counterparts, that can be highlighted in the reduced amplitude and increased frequency of torque pulsation, lower dc-link current harmonic of the multiphase drive system, lower power distribution per phase, and increased overall system reliability [2], [3]. These advantages enable multiphase drives to find a place in applications where safety and fault tolerance are of prime importance.

Among the numerous multiphase machine possibilities, sixphase machines are one of the most widely discussed multiphase topologies due to their modular three-phase structure which allows adaptation of existing three-phase technology. Depending on the spatial phase-shift between the two three-phase windings, six-phase machines can be categorized into symmetrical and asymmetrical configurations with $60^{\circ}$ and $30^{\circ}$ spatial phase-angles, respectively. Symmetrical six-phase machines, with isolated neutral points, have been investigated in several studies and found various industrial applications [4], [5].

Numerous MV converter topologies for high-power industrial drives have been investigated since the mid-1980s [6]-[9]. Among them, various multilevel converter topologies have established themselves as a mature technology which has been favored by MV drives manufacturers, such as the neutral-pointclamped converter [10], flying-capacitor converter [11], and cascaded H-bridge converter [12]. Recently, the commercialization of the modular multilevel converter (MMC) as a standard converter interface in the high-voltage directcurrent transmission field has offered new possibilities and options regarding its utilization as an MV machine drive based on its outstanding features in addition to the shortcomings of the mentioned state-of-the-art multilevel topologies [13]. The penetration of the MMC into the MV adjustable-speed drives market is still limited at this stage. The main reason is that the MMC-fed adjustable-speed drive suffers from power imbalance problems between the upper and lower arms when operating at high torque and low speed, where the voltage fluctuation of the MMC floating capacitors is directly proportional to the magnitude of the output current, while inversely proportional to the operating frequency.

Several studies have been directed towards restraining the inherent problem of wide voltage fluctuation of the MMC floating capacitors when feeding MV drives at low operating frequencies. Most of these studies inject a high-frequency circulating current into the MMC phase arms, while injecting the same frequency harmonic into the converter output common-mode $(\mathrm{CM})$ voltage. This allows the sub-module (SM) capacitors to be charged and discharged more frequently such that their voltage ripple is attenuated [14]-[16]. Although this compensation approach limits capacitor voltage variation at low motor speeds, the $\mathrm{CM}$ voltage introduced at the motor terminals has a negative impact on both motor insulation and bearings, that harms the motor and shortens its lifetime. Also, the high magnitude of the high-frequency arm currents significantly increases current stress, and consequently power loss, which necessitates overrating the current capacity of the switching devices. 
Other studies addressed MMC low-frequency operation through new control methods [17], operation modes [18], modulation techniques [19], or suggesting various modifications to the basic MMC structure [20]-[22].

Although most approaches introduced in the literature satisfactorily suppress the SM capacitor voltage-ripple at low operating frequencies, all are still unable to drive a machine at full-load torque from a stand-still condition. Additionally, no detailed verification and results have been demonstrated for continuous low-frequency operation of high-power machines within multi-megawatt drive systems.

This paper presents an MMC configuration for symmetrical six-phase machines, as a reliable solution to overcome the mentioned limitations of MMC-fed variable-speed drives. With the inherent spatial opposition in symmetrical machine windings, the ripple-power pulsates in an anti-phase manner in MMC phase arms feeding opposite machine windings. That is, the proposed configuration inserts bidirectional power transfer channels between each two adjacent-arm SMs of opposite MMC phase-legs, allowing the fundamental ripple-power to be decoupled. The ripple-power decoupling results in even redistribution of capacitive energy stored in the MMC arms, which eliminates a wide variation of capacitor voltage-ripple, independent of the operating frequency. Therefore, the proposed approach enables the MMC to drive a variable-speed machine at full load torque over the whole speed range, even at stand-still. Although the proposed configuration adds extra hardware to the basic MMC structure, it significantly reduces the sizing requirement of the SM capacitance, and hence, the energy stored in the MMC system. Also, the control of the added power-decoupling modules is independent of the main control loops of the MMC system.

\section{RipPle ANALYSIS OF CONVENTIONAL MMC}

Symmetrical six-phase machines can be supplied by a dual three-phase MMC to generate two sets of three-phase voltages with $60^{\circ}$ electrical-degrees phase-shift, as illustrated in the winding and phasor diagram of Fig. 1. Then each three-phase MMC is controlled independently to generate a multi-levelbased three-phase voltage-set which can be applied across either three-phase group of machine windings. Each MMC is composed of three-phase legs, each formed by two arms connected in series through arm inductors $L_{f}$. Each arm consists of $N$ series-connected SMs, while the SM consists of a halfbridge (HB) cell with a dc capacitor of an equivalent capacitance $C$ and voltage rating $V_{c}$, as shown in Fig. 2 .

A general form for symmetrical six-phase voltages generated by a six-phase $\mathrm{MMC}$ is:

$$
\begin{aligned}
v_{j o}=V_{o} \cos \left(\omega t-\frac{\pi}{3} k\right), k & =0,2,4 \text { for } j=a, b, c \\
\text { and } k & =1,3,5 \text { for } j=x, y, z
\end{aligned}
$$

where $\omega$ is the output angular frequency, while $V_{o}$ is the magnitude of the output phase-voltage which is bounded by the modulation index $M$ and the voltage of the input source $V_{d c}$, as shown in (2).

$$
V_{o}=\frac{1}{2} M V_{d c}
$$

Similarly, a general form for the six-phase currents passing through the symmetrical machine windings is:

$$
\begin{aligned}
i_{j}=I_{o} \cos \left(\omega t-\frac{\pi}{3} k-\emptyset\right), k & =0,2,4 \text { for } j=a, b, c \\
\text { and } k & =1,3,5 \text { for } j=x, y, z
\end{aligned}
$$

where $I_{o}$ is the magnitude of the machine current and $\varnothing$ is the power-factor angle of the machine.

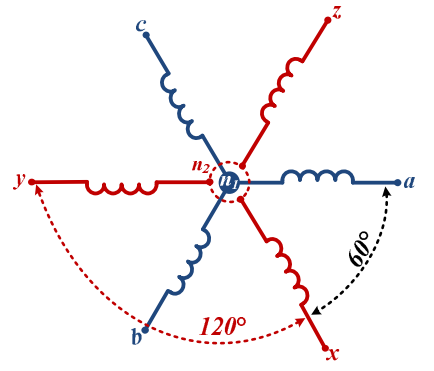

(a)

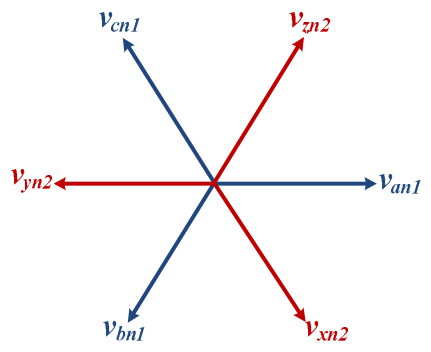

(b)
Fig. 1 Symmetrical six-phase machine. (a) Winding diagram and (b) phasor diagram of machine phase-voltages.

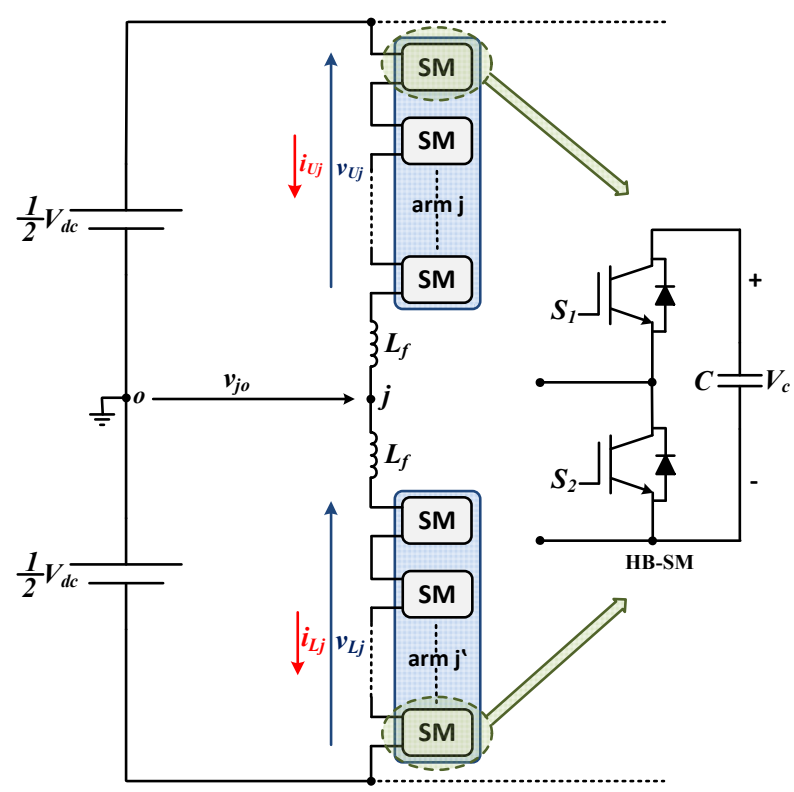

Fig. 2 Circuit diagram of an MMC phase-leg using HB-SMs.

The reference voltages for the MMC arms in addition to the arm currents are given by (4) and (5), respectively, with the subscripts $U$ and $L$ referring to the corresponding 'upper' and 'lower' arm.

$$
\begin{aligned}
& \left\{\begin{array}{l}
v_{U j}=\frac{1}{2} V_{d c}-v_{j o} \\
v_{L j}=\frac{1}{2} V_{d c}+v_{j o}
\end{array}\right. \\
& \left\{\begin{array}{l}
i_{U j}=i_{c m j}+\frac{1}{2} i_{j} \\
i_{L j}=i_{c m j}-\frac{1}{2} i_{j}
\end{array}\right.
\end{aligned}
$$

where $i_{c m} j$ is the CM current which can be referred to as the circulating current. With appropriate even-order harmonic suppression control [23], the CM current can be considered as only a dc component which can be calculated for a six-phase MMC through lossless power balance between the dc input and ac output as shown in (6), where $I_{d c}$ is the dc input current of the six-phase MMC.

$$
i_{c m j}=\frac{I_{d c}}{6}=\frac{M I_{o} \cos \emptyset}{4}
$$


The instantaneous power input to each MMC arm is given by the product of the arm voltage (4) and the corresponding arm current (5) as follows.

$$
\left\{\begin{array}{l}
p_{U j}=v_{U j} i_{U j} \\
p_{L j}=v_{L j} i_{L j}
\end{array}\right.
$$

Substituting (1)-(6) into (7) yields,

$$
\begin{aligned}
& p_{U j}=\frac{V_{d c} I_{o}}{4}\left[\cos \left(\omega t+\theta_{j}-\emptyset\right)\right. \\
& -\frac{M^{2}}{2} \cos (\varnothing) \cos \left(\omega t+\theta_{j}\right) \\
& \left.-\frac{M}{2} \cos \left[2\left(\omega t+\theta_{j}\right)-\emptyset\right]\right] \\
& p_{L j}=\frac{V_{d c} I_{o}}{4}\left[-\cos \left(\omega t+\theta_{j}-\emptyset\right)\right. \\
& +\frac{M^{2}}{2} \cos (\varnothing) \cos \left(\omega t+\theta_{j}\right) \\
& \left.-\frac{M}{2} \cos \left[2\left(\omega t+\theta_{j}\right)-\emptyset\right]\right]
\end{aligned}
$$

From (8), the instantaneous power in the upper and lower arms can be categorized into a CM component, $p_{c m}$, which is in phase in both arms, and a differential-mode (DM) component, $p_{d m}$, which appears in anti-phase in the upper and lower arms. The CM component alternates at twice the line frequency, and is a direct consequence of the active power at the dc-side being constant, whereas the phase-power at the acside pulsates at twice the line frequency. The DM component alternates with the fundamental line-frequency, and represents the power which is circulated back and forth internally between the upper and lower arms of the same leg. Accordingly,

$$
\begin{gathered}
p_{c m}=-\frac{V_{d c} I_{o} M}{8} \cos \left[2\left(\omega t+\theta_{j}\right)-\emptyset\right] \\
p_{d m}=\frac{V_{d c} I_{o}}{8} \sqrt{4+\cos ^{2}(\varnothing)\left(M^{4}-4 M^{2}\right)} \cos \left(\omega t+\theta_{j}-\gamma\right) \\
\gamma=\emptyset+\tan ^{-1} \frac{M^{2} \tan \emptyset \cos ^{2} \emptyset}{2-M^{2} \cos ^{2} \emptyset}
\end{gathered}
$$

Similarly, the energy variation of the arms can be classified into a CM component, $w_{c m}$, and a DM component, $w_{d m}$, as given by (11).

$$
\begin{aligned}
& w_{U j}=w_{c m}+w_{d m} \\
& w_{L j}=w_{c m}-w_{d m}
\end{aligned}
$$

Integrating (9) yields both energy-variation components as follows.

$$
\begin{gathered}
w_{c m}=-\frac{V_{d c} I_{o} M}{16 \omega} \sin \left[2\left(\omega t+\theta_{j}\right)-\emptyset\right] \\
w_{d m}=\frac{V_{d c} I_{o}}{8 \omega} \sqrt{4+\cos ^{2}(\varnothing)\left(M^{4}-4 M^{2}\right)} \sin \left(\omega t+\theta_{j}-\gamma\right)
\end{gathered}
$$

Due to the dual frequency alternation of the capacitive energy stored in the MMC arms, capacitor voltage-fluctuations have both $\mathrm{CM}$ and $\mathrm{DM}$ voltage-ripple components alternating at twice the fundamental frequency and at the fundamental frequency, respectively, as demonstrated by (13).

$$
\begin{array}{r}
\Delta v_{c_{U j}}=-\frac{\Delta V_{c_{c m}}}{2} \sin \left[2\left(\omega t+\theta_{j}\right)-\emptyset\right] \\
+\frac{\Delta V_{c_{d m}}}{2} \sin \left(\omega t+\theta_{j}-\gamma\right) \\
\begin{aligned}
\Delta v_{c_{L j}}=-\frac{\Delta V_{c_{c m}}}{2} \sin \left[2\left(\omega t+\theta_{j}\right)-\emptyset\right] \\
-\frac{\Delta V_{c_{d m}}}{2} \sin \left(\omega t+\theta_{j}-\gamma\right)
\end{aligned}
\end{array}
$$

where $\Delta v_{c_{U j}}$ and $\Delta v_{c_{L j}}$ are the capacitor voltage-variation of SMs in the upper and lower MMC arms, respectively, while $\Delta V_{c_{c m}}$ and $\Delta V_{c_{d m}}$ are the absolute values of the peak-to-peak variation of the CM and DM components of the SM capacitor voltage-ripple. That is, the variation of the arm stored-energy is managed by the capacitors of the series-connected SMs in each arm as shown by (14).

$$
\Delta W=\frac{N C}{2}\left(V^{2}{ }_{c \max }-V^{2}{ }_{c \min }\right)=N C V_{c} \Delta V_{c}
$$

where $\Delta W$ and $\Delta V_{c}$ are the peak-to-peak variation of the arm stored-energy and capacitor voltage-ripple of each SM, respectively.

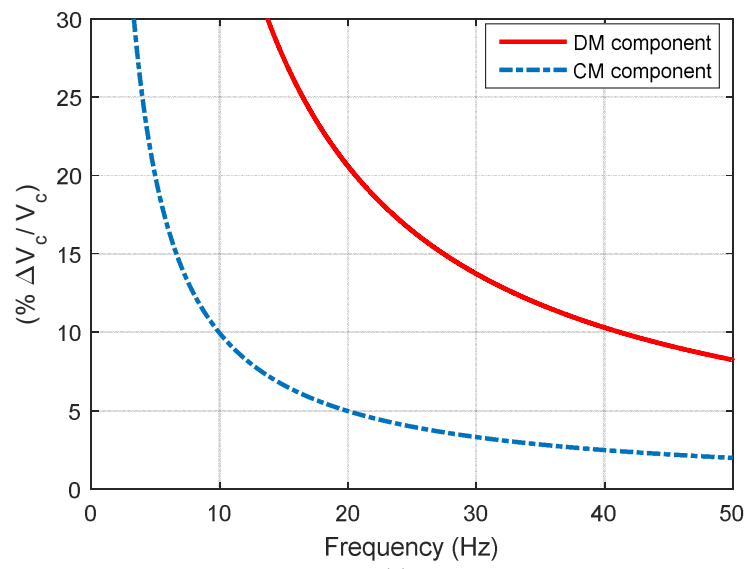

(a)

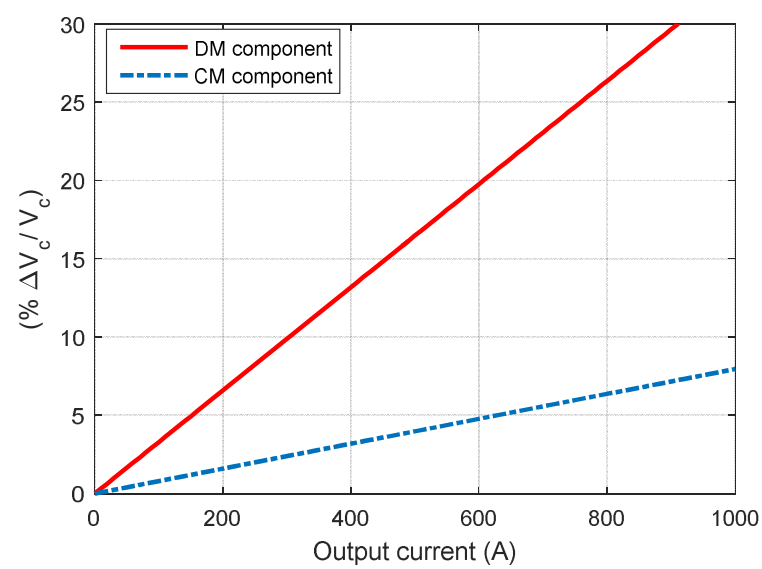

(b)

Fig. 3 Normalized components of capacitor voltage-ripple, with $V_{c}=2.5 \mathrm{kV}, C$ $=3 \mathrm{mF}, M=0.75$, and $\varnothing=25^{\circ}$, at: (a) variable frequency and constant output current $\left(I_{o}=500 \mathrm{~A}\right)$ and $(\mathrm{b})$ variable output current and constant frequency $(f=$ $25 \mathrm{~Hz})$. 


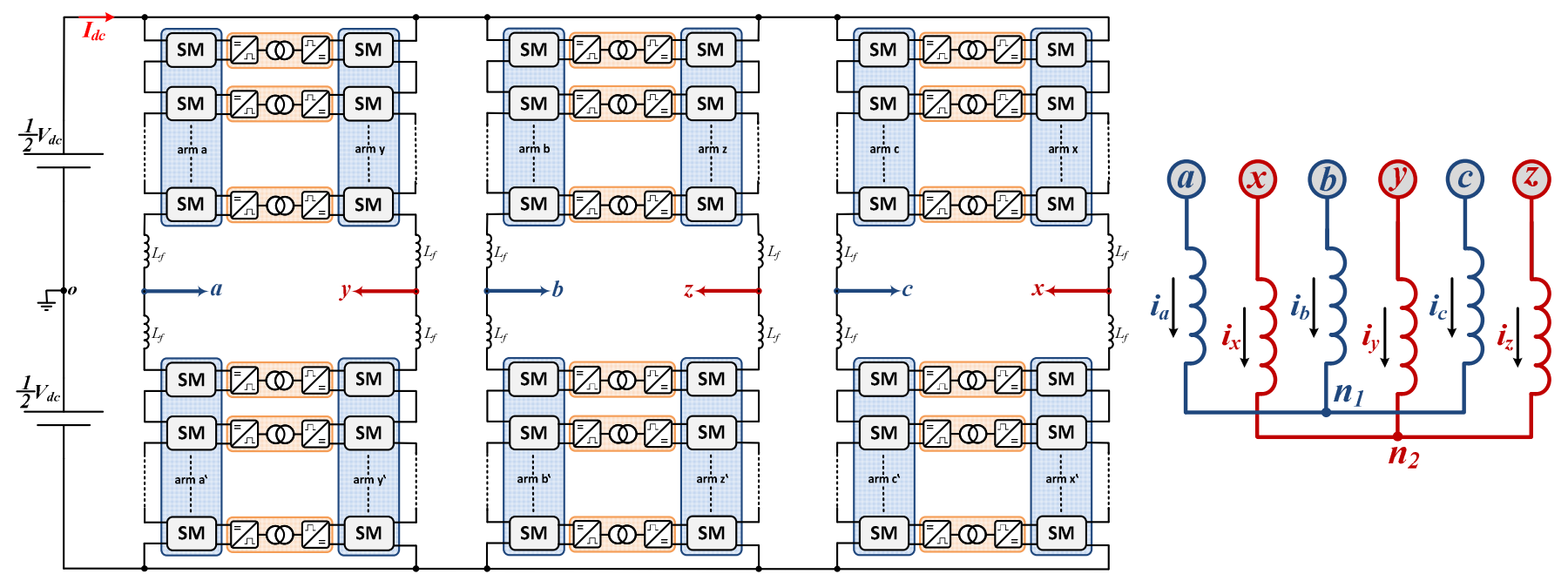

Fig. 4 Circuit diagram of the proposed MMC configuration for symmetrical six-phase machines.

Since the average voltage across each SM capacitor is $V_{c}=$ $V_{d c} / N$, the magnitude of the peak-to-peak capacitor voltageripple due to CM and DM components, from (12) and (14) is:

$$
\begin{gathered}
\Delta V_{c_{c m}}=\frac{I_{o} M}{8 \omega C} \\
\Delta V_{c_{d m}}=\frac{I_{o}}{4 \omega C} \sqrt{4+\cos ^{2}(\varnothing)\left(M^{4}-4 M^{2}\right)}
\end{gathered}
$$

From (15a), the CM component has slight influence on capacitor voltage-ripple, where in variable-speed drives with Volt/Hertz control, the ratio $M / \omega$ is constant, while the load current $I_{o}$ is also invariant. On the other side, when the operating frequency $\omega$ is reduced in (15b), while the ratio $M / \omega$ is kept constant for motor constant torque requirements, the DM component of the capacitor voltage-ripple increases. Furthermore, the direct proportionality of capacitor voltageripple to variation in output current at a constant operating frequency is much higher in the DM component, compared to the CM component. The significant influence of the DM component on the capacitor voltage-ripple can be emphasized as demonstrated by Fig. 3, where the normalized peak-to-peak capacitor voltage-ripple for both $\mathrm{CM}$ and DM components is illustrated with operating frequency variation in Fig. 3a, and with output current variation in Fig. $3 b$.

\section{PRoposed MMC CONFIGURATION FOR SYMMETRICAL SIX-PHASE MACHINES}

The spatial opposition between each two machine winding of the symmetrical six-phase machine implies the DM power component pulsates in an anti-phase profile in the corresponding oppositely modulated phase-arms. The proposed MMC configuration decouples the DM power component by creating a physical channel for bidirectional power flow between opposite MMC arms feeding two machine windings with spatial phase-opposition. Since the energy stored in each MMC arm is equally shared by all SMs subsisted by this arm through an individual capacitor voltage balancing technique, a modular implementation of energy exchange between oppositely modulated arms is achieved by inserting highfrequency (HF) transformer-based dc-dc converter modules between each two adjacent SMs. This allows a redistribution of the stored energy among each adjacent pair of SMs by transferring the DM power component from the SM with arm current charging its capacitor into the opposite SM with a discharging current-direction. With such a bidirectional powertransfer scheme, the DM component of energy variation is cancelled, while the CM component is evenly distributed among the SMs. This approach is illustrated in Fig. 4, where the proposed MMC configuration for a symmetrical six-phase machine is shown.

\section{A. Isolated DC-DC Converter Modules}

The concept of dc-dc conversion via an HF transformer has been proposed as a candidate to achieve high power-density dcdc conversion with the distinct advantages of bidirectional power flow, HF galvanic isolation, high efficiency, low weight and size, and soft switching of the employed semiconductor devices [24]. The phase-shift dual-bridge converter is the common implementation of $\mathrm{HF}$ transformer based dc-dc converters, in which two bridges are interfaced through an HF transformer, phase shifted from each other to control the power transfer between the two dc-voltage buses. The most common configurations of phase-shift dual-bridge dc-dc converter are the phase-shift dual active-bridge and dual half-bridge (DHB) converters [25]. In this paper, the phase-shift DHB is employed as a dc-dc energy-exchange module that achieves energy balancing for MMC SMs. A detailed circuit diagram for one of the front-to-front SMs of the proposed MMC configuration shown in Fig. 4 is elucidated in Fig. 5, where two HB-SMs are interfaced through a DHB dc-dc converter.

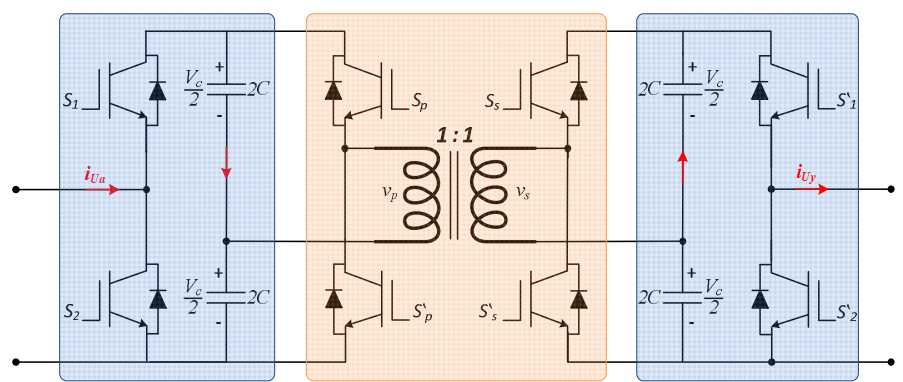

Fig. 5 DHB configuration for two adjacent HB-SMs of the proposed MMC topology. 
The DHB converter consists of two voltage-source HB dc-ac inverters coupled through an $\mathrm{HF}$ isolation transformer. The transformer turns ratio is unity since the dc-dc conversion occurs between bridge sides at the same voltage level equal to the average voltage of MMC SM capacitors. Both HB inverters generate fixed-frequency square-wave voltages with a constant duty ratio of $50 \%$, applied to both sides of the transformer. The configuration of the DHB necessitates a center-tap connection to each SM capacitor as shown in Fig. 5.

\section{B. DHB Control}

The phase-shift angle, $\sigma$, between the two square-wave voltages of the DHB converter determines the amount of power transferred from the leading bridge to the lagging bridge. With unity transformer turns-ratio and equal voltage levels, $V_{c}$, at both bridge sides, the DHB power is expressed as [26]:

$$
P=\frac{V_{c}^{2} \sigma(\pi-|\sigma|)}{8 \pi^{2} f_{h} L}
$$

where $L$ is the transformer leakage inductance and $f_{h}$ is the DHB switching frequency.

The DM component of SM capacitor voltage-ripple is controlled at both DHB sides to follow a zero reference value through a proportional integral (PI) controller. The capacitor voltage-ripple is obtained from the SM capacitor voltage using a high-pass filter as shown in the block diagram of Fig. 6 . Depending on the voltage-ripple error, the PI controller outputs a suitable phase-shift angle to control the power flow between both sides of the DHB, allowing the DM component of pulsating power to be transferred from the SM interfaced with the leading bridge to the adjacent SM interfaced with the lagging bridge, to counterbalance the same power component.

Referring to Fig. 5, the front-to-front SMs are assumed in the upper arms of phase-legs $a$ and $y$. Forward power-transfer, designated as a positive phase-shift, occurs during the positive half-cycle of the machine current $i_{a}$. In this case, the primaryside $\mathrm{HB}$ converter is the leading bridge while the secondaryside HB converter is the lagging bridge. The same power transfer action is repeated during the negative half-cycle of the machine current, but with reversal of leading and lagging bridge sides.

\section{Capacitor Voltage-Ripple}

The DM component of the pulsating power is zero for each front-to-front SM, while the CM component is unchanged. Therefore, the voltage ripple across SM capacitors of the proposed MMC configuration exhibits a second-order sinusoidal fluctuation, as given by (17) with an absolute peakto-peak value equal to the CM component as in (18).

$$
\begin{aligned}
& \Delta v_{c_{U j} \text { proposed }}=\Delta v_{c_{L j} \text { proposed }} \\
&=- \frac{I_{o} M}{16 \omega C} \sin \left[2\left(\omega t+\theta_{j}\right)-\emptyset\right] \\
& \Delta V_{c_{\text {proposed }}}=\Delta V_{c_{c m}}=\frac{I_{o} M}{8 \omega C}
\end{aligned}
$$

Based on (17), the SM capacitor voltage-ripple in the proposed MMC configuration inherits the same behavior as the $\mathrm{CM}$ component which is found to have the least influence on capacitor voltage-ripple, as shown previously in Fig. 3.

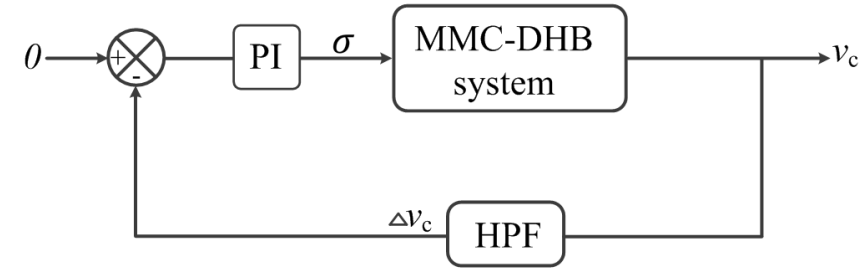

Fig. 6 DHB control scheme.

\section{Operation at Zero Motor-Speed/frequency}

One of the salient advantages of the proposed MMC configuration is that it has the capability of starting a machine at full-load torque from a stand-still condition with a ripple-free SM capacitor voltage-profile. That is, at near zero frequency, the power drawn at the output side will be near dc power, and therefore, the $\mathrm{CM}$ component of the pulsating power in $\mathrm{MMC}$ arms will be zero. Also, with unidirectional transfer of the dc DM power component between opposite SMs in both the upper and lower arms, the net pulsating power in different arms will be zero. In this case, the capacitive energy stored in the MMC arms will be redistributed evenly at a constant level among all SMs, resulting in a constant voltage across each SM capacitor.

\section{Simulation AND EXPERIMENTAL RESUlts}

The effectiveness of the proposed MMC configuration is verified via a $20 \mathrm{MW}$ MATLAB/SIMULINK model in addition to a $4 \mathrm{~kW}$ scaled-down laboratory prototype, both investigated during different scenarios. Parameters and operating conditions are listed in Table I for both simulation and experimentation. An $R L$ load is used to examine the steady-state performance of the proposed MMC and to assess the SM capacitor voltageripple at different operating frequencies. To maintain constant rated output current, the output voltage is reduced in accordance to operating frequency reduction, while the load resistance is varied linearly with the operating frequency change.

TABLE I

PARAMETERS FOR SIMULATION AND EXPERIMENTAL STUDIES

\begin{tabular}{||l|c|c||}
\hline \hline Parameter & Simulation & Experiment \\
\hline Number of SMs per arm $(N)$ & 10 & 3 \\
\hline Input dc voltage $\left(V_{d c}\right)$ & $25 \mathrm{kV}$ & $300 \mathrm{~V}$ \\
\hline Nominal SM capacitor voltage $\left(V_{c}\right)$ & $2.5 \mathrm{kV}$ & $100 \mathrm{~V}$ \\
\hline Rated active power & $20 \mathrm{MW}$ & $4 \mathrm{~kW}$ \\
\hline Rated current magnitude $\left(I_{o}\right)$ & $655 \mathrm{~A}$ & $10 \mathrm{~A}$ \\
\hline Fundamental output frequency $\left(f_{o}\right)$ & $50 \mathrm{~Hz}$ & $50 \mathrm{~Hz}$ \\
\hline Carrier frequency of MMC $\left(f_{c}\right)$ & $2 \mathrm{kHz}$ & $2 \mathrm{kHz}$ \\
\hline Switching frequency of DHB $\left(f_{h}\right)$ & $10 \mathrm{kHz}$ & $10 \mathrm{kHz}$ \\
\hline Load resistance $(R)$ & $15.5 \times \frac{f_{o}}{50} \Omega$ & $11 \times \frac{f_{o}}{50} \Omega$ \\
\hline Load inductance $(L)$ & $24 \mathrm{mH}$ & $20 \mathrm{mH}$ \\
\hline Arm inductance $\left(L_{f}\right)$ & $2 \mathrm{mH}$ & $2.8 \mathrm{mH}$ \\
\hline Equivalent SM series capacitance $(C)$ & $2 \mathrm{mF}$ & $1.1 \mathrm{mF}$ \\
\hline \hline
\end{tabular}

\section{A. Simulation Results}

Fig. 7 shows the fundamental waveforms for 20 MW steadystate operation of the proposed MMC configuration at both rated output current and frequency, as defined in Table I. The modulation index is set to 0.9 to generate an $11.3 \mathrm{kV}$ output phase-voltage across the six-phase machine windings. The six- 
phase machine currents are of high-quality with a current peak equal to the rated value of $655 \mathrm{~A}$. The currents in upper and lower arms are controlled to suppress both second- and fourthorder harmonic components to give a nearly-constant CM current as assumed in the mathematical analysis in Section II. The recorded peak arm currents are $510 \mathrm{~A}$, while the average $\mathrm{CM}$ current is $135 \mathrm{~A}$.
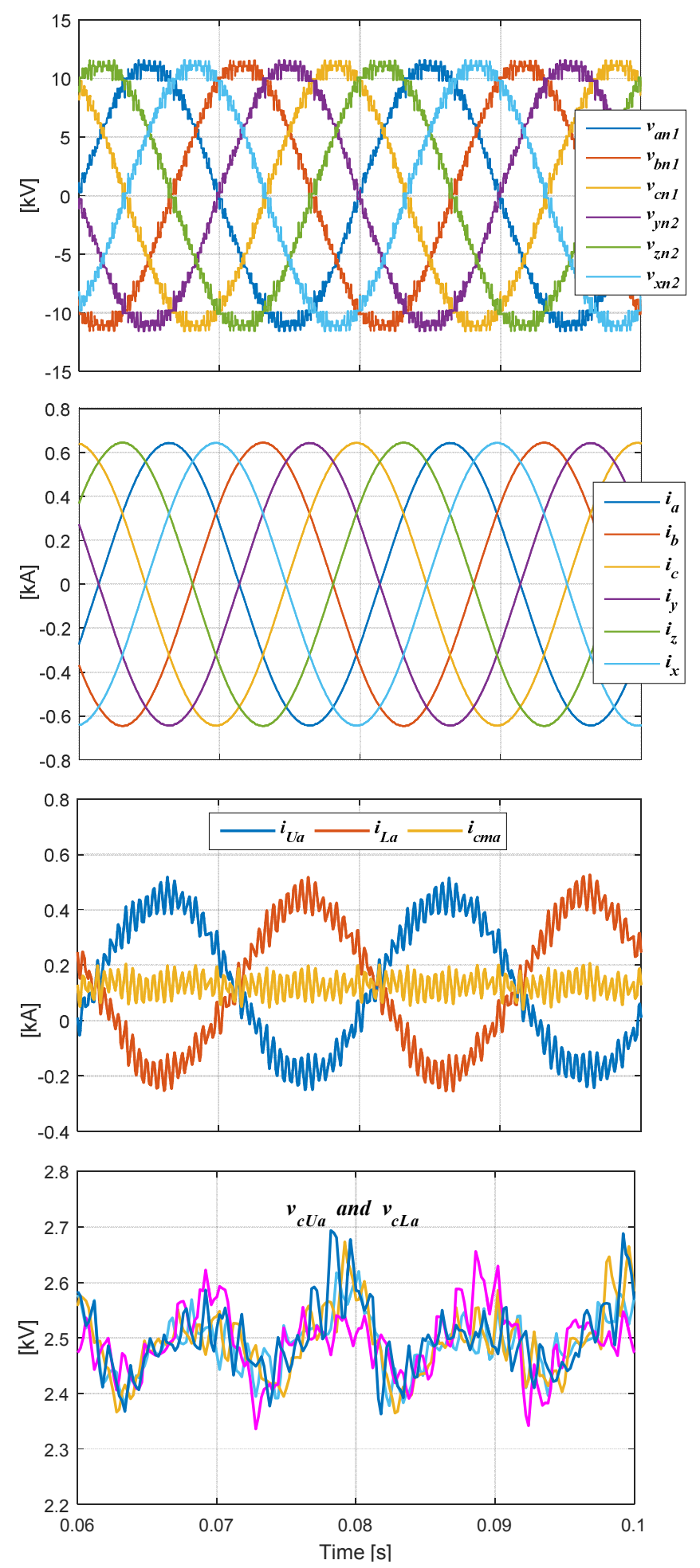

Fig. 7 Simulation waveforms of the proposed MMC configuration at $50 \mathrm{~Hz}$.
The capacitor voltage-ripple alternates at twice the line frequency with $\pm 6 \%$ peak-to-peak voltage-ripple, using an equivalent $\mathrm{SM}$ capacitance of $2 \mathrm{mF}$.

To assess the reduction in SM capacitor voltage-ripple, Fig. 8 shows the capacitor voltage-variation of the proposed MMC configuration with the deactivation of the switching signals of the DHB modules during the simulation mid-period, at low operating frequencies. With energy-balancing deactivation, capacitor voltage-ripple significantly increases especially when the operating frequency is reduced. While the voltage-ripple of SM capacitors is near constant at $\pm 5 \%$ at the three-different frequencies when the DHB modules are operational, the voltage ripple after DHB deactivation is $\pm 33 \%, \pm 50 \%$, and $\pm 66 \%$ at $10 \mathrm{~Hz}, 5 \mathrm{~Hz}$, and $1 \mathrm{~Hz}$, respectively. Although the latter voltageripple percentages are impractically high, they highlight the significant reduction in the sizing of capacitive storage-element which could be achieved by counter-balancing the ripple power between adjacent SMs of the MMC legs through the incorporation of DHB modules.

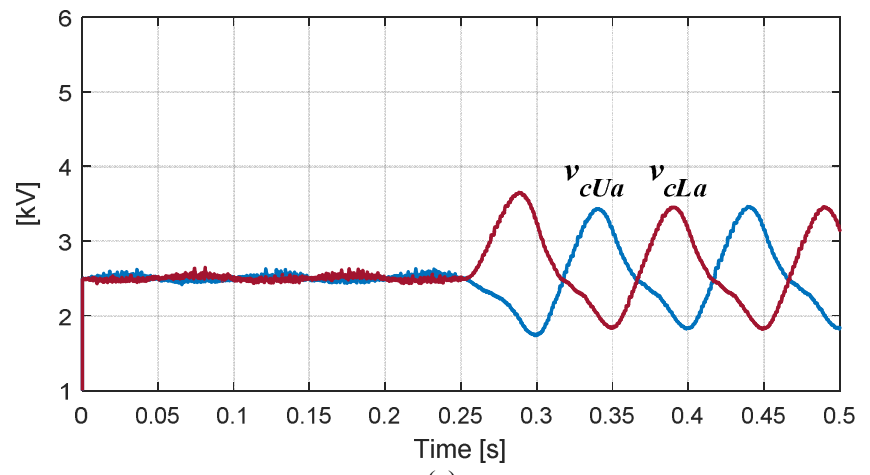

(a)

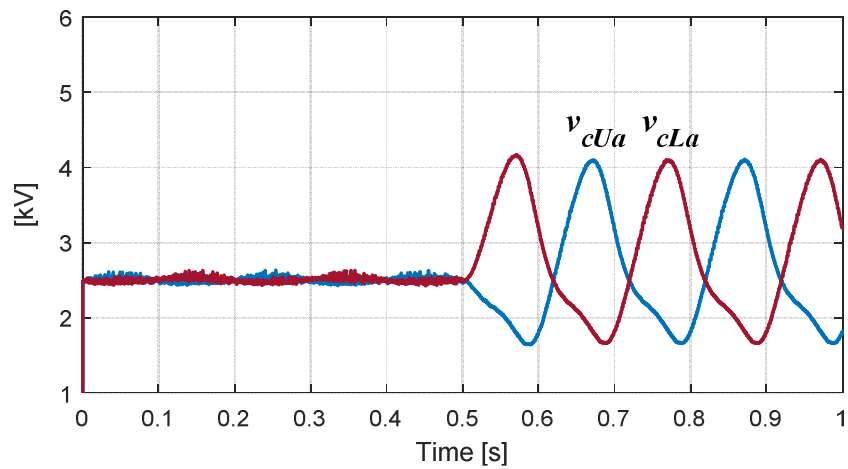

(b)

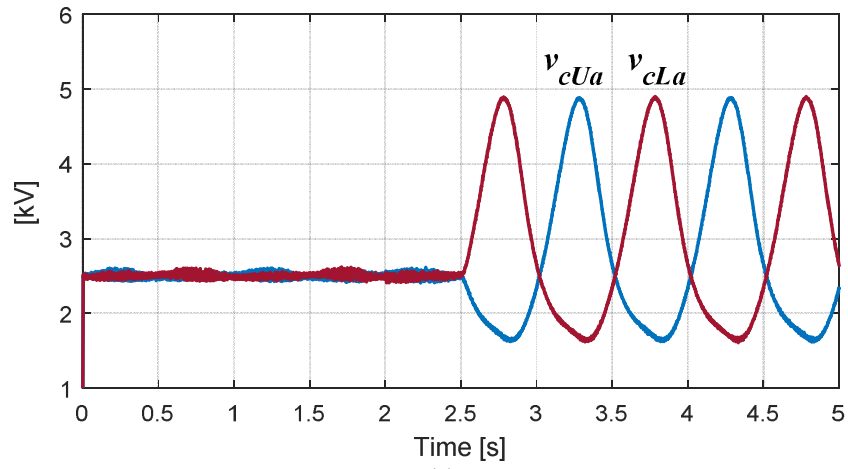

(c)

Fig. 8 SM capacitor voltage-variation with deactivation of DHB modules at the mid time of simulation at (a) $10 \mathrm{~Hz}$, (b) $5 \mathrm{~Hz}$, and (c) $1 \mathrm{~Hz}$. 


\section{B. Experimental Results}

The experimental arrangement is shown in Fig. 9, where adjacent SMs are interfaced through a DHB converter employing a nanocrystalline core HF transformer with a unity turns ratio. Fig. 10 shows the fundamental waveforms of $4 \mathrm{~kW}$ steady-state operation of the proposed MMC configuration at both rated current and frequency. The $60^{\circ}$ phase-shift between the two sets of phase-voltages and the $180^{\circ}$ phase-shift between adjacent MMC legs feeding machine windings with spatial opposition are shown, along with the corresponding machine currents. The phase voltages have three voltage-levels with a peak of half the dc input voltage, while the output currents are of high-quality with a magnitude of $10 \mathrm{~A}$. The SM capacitor voltage-ripple alternates at twice the fundamental frequency due to the CM component, with a peak-to-peak voltage ripple of $8 \mathrm{~V}( \pm 4 \%)$. Since the experimental setup does not incorporate even-order harmonic suppression, the CM current includes second-order harmonics that clearly affects both arm current waveforms.

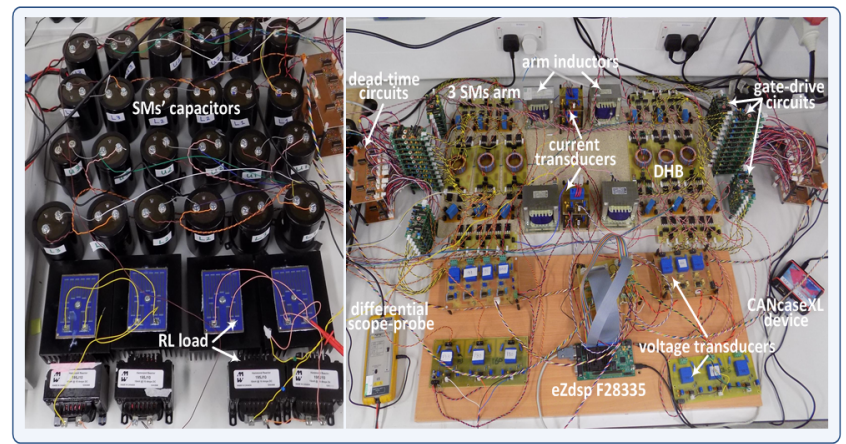

Fig. 9 Experimental setup.
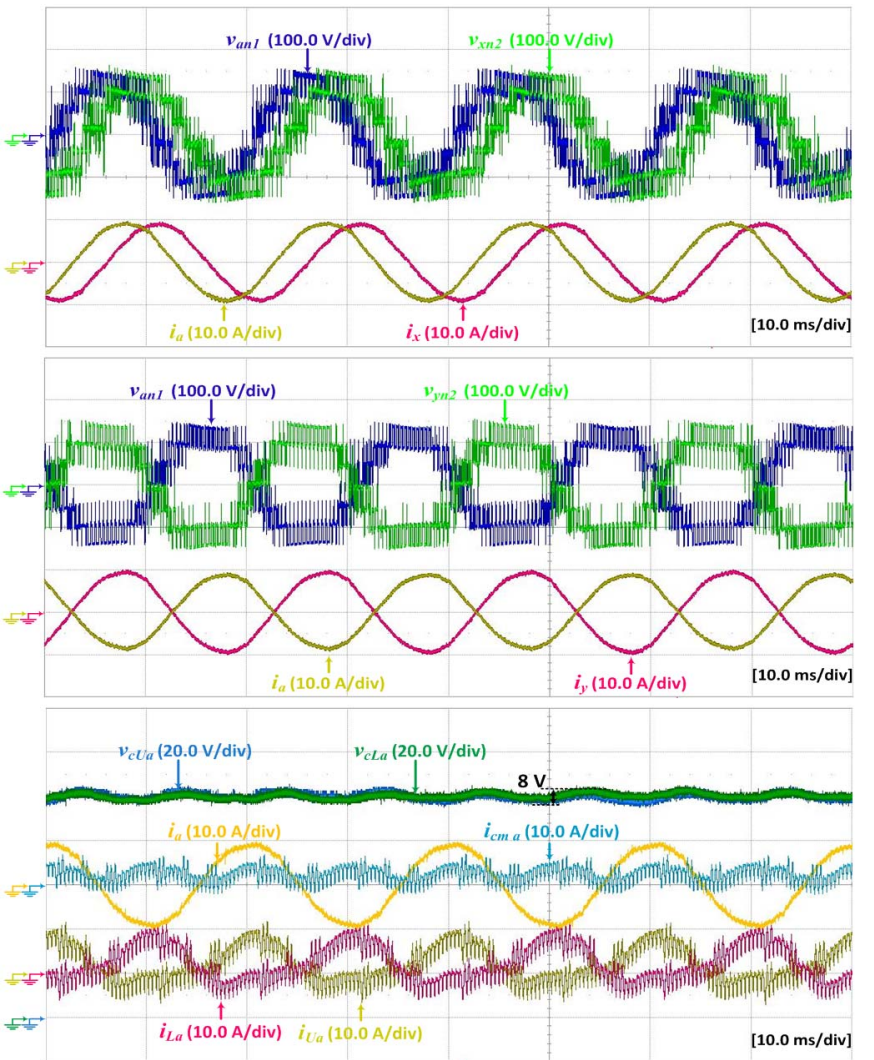

Fig. 10 Experimental waveforms of the proposed MMC configuration at $50 \mathrm{~Hz}$.
The switching waveforms of the phase-shift DHB converter in Fig. 11, show the voltages across both transformer sides along with the transformer current during both forward and reverse power flow. Both primary- and secondary-side voltages are square waveforms with a constant duty cycle of $50 \%$ and near $50 \mathrm{~V}$ peak, which is half the nominal voltage across each SM capacitor.

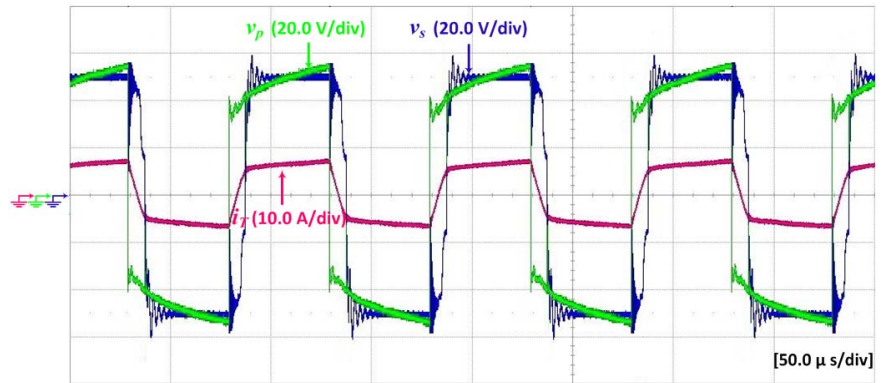

(a)

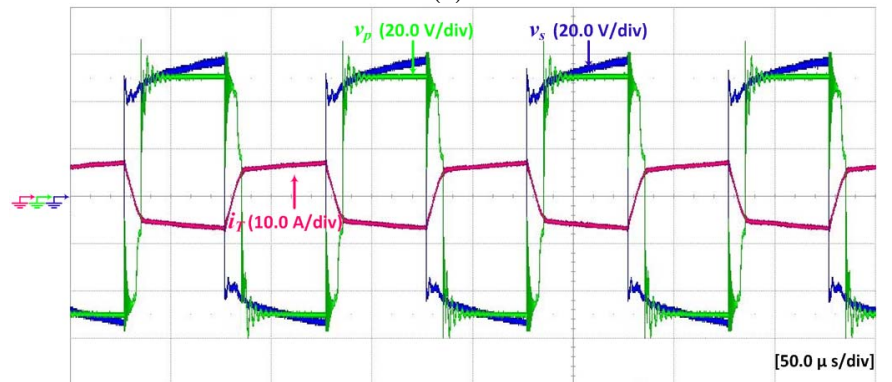

(b)

Fig. 11 DHB switching waveforms at (a) positive and (b) negative phase-shift angles.

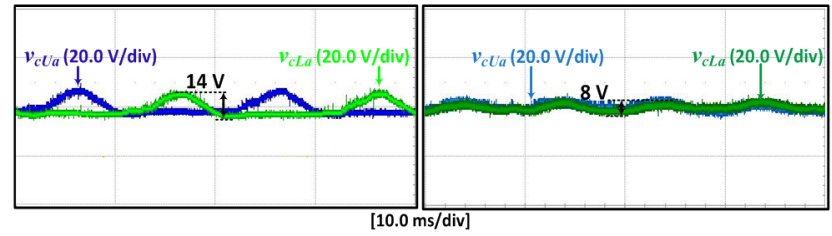

(a)

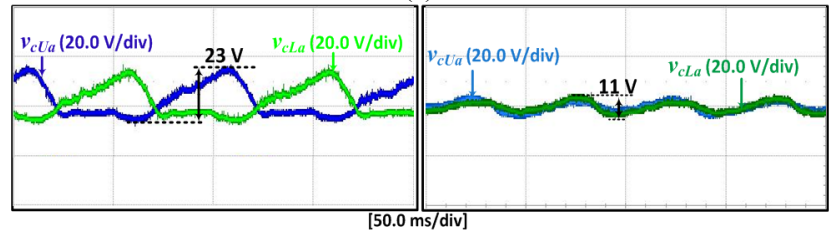

(b)

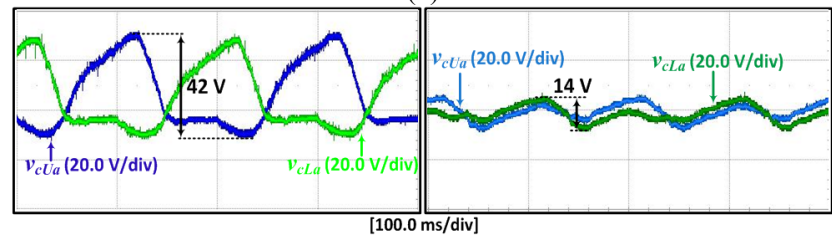

(c)

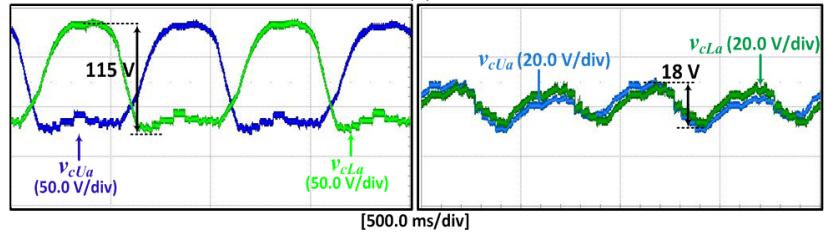

(d)

Fig. 12 SM capacitor voltage-ripple with DHB modules deactivated (left) and activated (right) at (a) $50 \mathrm{~Hz}$, (b) $10 \mathrm{~Hz}$, (c) $5 \mathrm{~Hz}$, and (d) $1 \mathrm{~Hz}$. 
Fig. 12 exhibits the significant influence of the DHB modules incorporation in the proposed MMC configuration, where the SM capacitor voltage-ripple is shown before and after DHB activation at different operating frequencies. From left to right, the capacitor voltage-ripple is shown with the DHB modules deactivated and operational, respectively. From Fig. 12a to 12d, the percentage reduction in the SM capacitor voltage-ripple, due to the activation of DHB modules, is recorded as $42 \%, 52 \%$, $66 \%$, and $84 \%$ at $50 \mathrm{~Hz}, 10 \mathrm{~Hz}, 5 \mathrm{~Hz}$, and $1 \mathrm{~Hz}$, respectively.

\section{CONCLUSION}

A novel MMC configuration for MV high-power six-phase drives, incorporating symmetrical winding-connection, has been presented. The configuration is characterized by the insertion of DHB converter modules between adjacent SMs subsisted by two MMC phase-legs operated with out-of-phase modulation, as a consequence of feeding two machine windings in spatial phase-opposition. Based on the fact that the fundamental ripple-power of adjacent SMs in the proposed MMC configuration alternates in anti-phase, the DHB modules can decouple this ripple-power component, allowing the capacitive stored-energy to be evenly distributed among all SMs. Thus the proposed configuration offers a significant reduction in SM capacitance since capacitor voltage fluctuation is suppressed, independent of the operating frequency. Further, the proposed MMC configuration ensures continuous operation of the symmetrical six-phase drive at any speed/torque condition, with the ability to drive multi-megawatt machines from stand-still at full-load torque. Theoretical deductions have been validated by simulations and experimentation, and are in agreement with theoretical analysis.

\section{REFERENCES}

[1] R. D. Klug, N. Klaassen, "High Power Medium Voltage DrivesInnovations, Portfolio, Trends," 2005 European Conference on Power Electronics and Applications, 11-14 September 2005.

[2] E. Levi, "Multiphase Electric Machines for Variable-Speed Applications," IEEE Trans. Ind. Electron, vol.55, no.5, pp.1893-1909, May 2008.

[3] E. Levi, R. Bojoi, F. Profumo, H. A. Toliyat and S. Williamson, "Multiphase induction motor drives-A technology status", IET Elect. Power Appl., vol. 1, no. 4, pp.489 -516 2007.

[4] M. S. Diab, A. A. Elserougi, A. S. Abdel-Khalik, A. M. Massoud and S. Ahmed, "A Nine-Switch-Converter-Based Integrated Motor Drive and Battery Charger System for EVs Using Symmetrical Six-Phase Machines," IEEE Trans. Ind. Electron., vol. 63, no. 9, pp. 5326-5335, Sept. 2016.

[5] R. Kianinezhad, B. Nahid-Mobarakeh, L. Baghli, F. Betin and G. A. Capolino, "Modeling and Control of Six-Phase Symmetrical Induction Machine Under Fault Condition Due to Open Phases," IEEE Trans. Ind. Electron, vol. 55, no. 5, pp. 1966-1977, May 2008.

[6] K. H. J. Chong, R. D. Klug, "High power medium voltage drives", Proc. Power Syst. Technol. PowerCon'04, pp. 658-664, 2004-Nov.-21-24.

[7] S. Bernet, "Recent developments of high power converters for industry and traction applications," IEEE Trans. Power Electron., vol. 15, no. 6, pp. 1102-1117, Nov. 2000.

[8] S. Rizzo and N. Zargari, "Medium voltage drives: what does the future hold?," The 4th International Power Electronics and Motion Control Conference, 2004. IPEMC 2004., Xi'an, 2004, pp. 82-89 Vol.1.

[9] D. Krug, M. Malinowski and S. Bernet, "Design and comparison of medium voltage multi-level converters for industry applications," IEEE Industry Applications Conference, 2004. 39th IAS Annual Meeting., 2004, pp. $781-790$ vol.2.

[10] A. Bendre, G. Venkataramanan, D. Rosene, and V. Srinivasan, "Modeling and design of a neutral-point voltage regulator for a three-level diode clamped inverter using multiple-carrier modulation," IEEE Trans. Ind. Electron., vol. 53, no. 3, pp. 718-726, Jun. 2006.

[11] I.-D. Kim, E.-C. Nho, H.-G. Kim, and J. S. Ko, "A generalized undeland snubber for flying capacitor multilevel inverter and converter," IEEE Trans. Ind. Electron., vol. 51, no. 6, pp. 1290-1296, Dec. 2004.

[12] S. Sirisukprasert, J. S. Lai, and T. H. Liu, "Novel cascaded multilevel converter drive system with minimum number of separated DC sources," in Proc. IEEE PESC, Vancouver, BC, Canada, Jun. 17-22, 2001, pp. $1346-1350$

[13] Maryam Saeedifard and Reza Iravani, "Dynamic performance of a modular multilevel back-to-back HVDC system," IEEE Trans. Power Del., vol. 25, no. 4, pp. 2903-2912, Oct. 2010.

[14] A. Antonopoulos, L. Angquist, S. Norrga, K. Ilves, L. Harnefors, H.-P. Nee, "Modular multilevel converter ac motor drives with constant torque from zero to nominal speed," IEEE Trans. Ind. Appl., vol. 50, no. 3, pp. 1982-1993, May/Jun. 2014.

[15] M. Hagiwara, I. Hasegawa, and H. Akagi, "Start-up and low-speed operation of an electric motor driven by a modular multilevel cascade inverter," IEEE Trans. Ind. Appl., vol. 49, no. 4, pp. 1556-1565, Jul./Aug. 2013.

[16] B. Li, S. Zhou, D. Xu, R. Yang, D. Xu, C. Buccella, and C. Cecati, "An improved circulating current injection method for modular multilevel converters in variable-speed drives" IEEE Trans. Ind. Electron., vol. 63, no. 11, pp. 7215-7225, Nov. 2016.

[17] A. Antonopoulos, L. Ängquist, L. Harnefors and H. P. Nee, "Optimal Selection of the Average Capacitor Voltage for Variable-Speed Drives with Modular Multilevel Converters," IEEE Trans. Power Electron, vol. 30, no. 1, pp. 227-234, Jan. 2015.

[18] Yang; B. Li; G. Wang; C. Cecati; S. Zhou; D. G. Xu; W. Yu, "Asymmetric mode control of MMC to suppress capacitor voltage ripples in low frequency low voltage condition," IEEE Trans. Power Electron., vol. 32, no. 6, pp. 4219-4230, June 2017.

[19] A. Mertens and J. Kucka, "Quasi Two-Level PWM Operation of an MMC Phase Leg with Reduced Module Capacitance," IEEE Trans. on Power Electron., vol. 31, no. 10, pp. 6765-6769, Oct. 2016.

[20] S. Du, B. Wu, K. Tian, N. R. Zargari and Z. Cheng, "An Active CrossConnected Modular Multilevel Converter (AC-MMC) for a MediumVoltage Motor Drive," IEEE Trans. Ind. Electronics, vol. 63, no. 8, pp. 4707-4717, Aug. 2016.

[21] S. Du; B. Wu; N. Zargari; Z. Cheng, "A Flying-Capacitor Modular Multilevel Converter (FC-MMC) for Medium-Voltage Motor Drive," IEEE Trans. Power Electron., vol. 32, no. 3, pp. 2081-2089, March 2017.

[22] L. Baruschka, D. Karwatzki, M. von Hofen and A. Mertens, "Low-speed drive operation of the modular multilevel converter Hexverter down to zero frequency," 2014 IEEE Energy Conversion Congress and Exposition (ECCE), Pittsburgh, PA, 2014, pp. 5407-5414.

[23] Z. Li, P. Wang, Z. Chu, H. Zhu, Y. Luo, and Y. Li, "An inner current suppressing method for modular multilevel converters," IEEE Trans. Power Electron., Vol. 28, No. 11, pp. 4873-4879, Nov. 2013.

[24] R. W. A. A. De Doncker, D. M. Divan and M. H. Kheraluwala, "A threephase soft-switched high-power-density DC/DC converter for high-power applications," in IEEE Trans. Ind. Applications, vol. 27, no. 1, pp. 63-73, $\mathrm{Jan} / \mathrm{Feb} 1991$

[25] S. Inoue and H. Akagi, "A bidirectional isolated DC-DC converter as a core circuit of the next-generation medium-voltage power conversion system," IEEE Trans. Power Electron., vol. 22, no. 2, pp. 535-542, Mar. 2007.

[26] F. Z. Peng, H. Li, G.-J. Su, and J. S. Lawler, "A new ZVS bidirectional dc-dc converter for fuel cell and battery application," IEEE Trans. Power Electron., vol. 19, no. 1, pp. 54-65, Jan. 2004. 